dustrie auszeichnet. Der Verfasser darf in diesem Sinne hier nochmals den Schluß seiner ersten Abhandlung über die "reziproken Salzpaare" zitieren:

"Wie für den Aufbau, so sind Gleichgewichtsuntersuchungen" auch für den Abbau der natürlichen Salzlager von wesentlicher Bedeutung. Die hochentwickelte und wirtschaftlich so bedeutsame Salzindustrie wird durch sie nur gewinnen und aus ihrem jetzigen Stadium empirisch festgestellter vorteilhafter Darstellungsweisen in eine Periode eines auf rein wissenschaftlichen Grundlagen ruhenden Betriebes eintreten."

Diese Stellungnahme M e y er hoffer $\mathbf{s}$ zu den rein empirischen Arbeitsmethoden der Kaliindustrie enthält die Antwort auf die Frage, in welcher Richtung die Entwicklung der technischen Verarbeitung der Kalirohsalze zu hochwertigen Fabrikaten gehen muß, wenn sie die Verbesserung ihrer Arbeitsmethoden in chemisch- und in wärmetechnischer Beziehung anstreben will. Unsere Aufgabe als Chemiker ist es, die Kaliindustrie aus dem Zustande einer emp/rischen Kochkunst in das Stadium einer mit dem Rüstzeug der modernen Wissenschaft arbeitenden, hochentwickelten Technik hinüberzuleiten. In den Untersuchungen $v$ a $n$ 't $H$ of $f \mathbf{s}$ ist uns der Weg zu diesem Ziele gewiesen.

[A. 176.]

\section{Bemerkungen zum Stereogramm des kristallinen Chlornatriums.}

\section{Von F. RINNE, Leipzig. ${ }^{\text {i }}$}

Der Aufforderung seitens der Schriftleitung, mich an der Ausgabe einer Sondernummer der Zeitschrift für angewandte Chemie zu Ebren von Prof. Dr. H. Precht zu beteiligen, folge ich sebr gern. Ich wähle zu dem Zwecke als Thema die feinbaulicbe Würdigung des Steinsalzes als eines Materials, dem der Jubilar bei seinen Studien stets ein besonders reges Interesse gewidmet hat.

1. Das Steinsalz ist das erste und damit klassische Beispiel einer erfolgreich vollzogenen Erkundung der stereochemischen Verhältnisse von Stoffen mittels Rontgenstrahlung.

Es setzt sich im Sinne der bekannten Fig. 1 aus atomionistischen Natrium- und Chlorbaugruppen zusammen. Die Hüllsphäre der $\mathrm{Na}$ triumionen hat man sich somit elektronenzählig entsprechend der des Neons, die der Chlorionen nach Art der Argonschale zu denken. Die Natriumkerne sind, soviel man weiß, untereinander gleich; die des Chlors sind isolopisch verschieden, insofern die Ionen $\mathrm{Cl}_{(35)}$ und $\mathrm{Cl}_{(37)}$ im Verhältnis von rund $75 \mathrm{zu} 25 \%$ am Kristall teilnebmen ${ }^{1}$ ). Da im Raumgitter des Chlornatriums an einem Punkte natürlich nur e in e Plejadenform des Chlors Platz hat, so muß man sich das Stereogramm des Steinsalzes nach Art der schematischen Fig. 2 aufgebaut denken, in $\operatorname{der} \mathrm{Cl}_{(35)}$ und $\mathrm{Cl}_{(37)}$ verteilt sind. Damit gewinnt dies Chlornatriumaggregat eine besondere feinbauliche, physikalisch-chemische Bedeutung: es stellt trotz seiner einfachen $\mathrm{NaCl}$-Formel eine eigene Art is omorpher Mischung dar.

Die drei Forderungen für das Bestehen des Isomorphismus: 1. chemische Analogie, 2. Fähigkeit des Zusammenkristallisierens und 3. ähnliche Kristallform, sind im vorliegenden Falle in extremer Art, also ideal, erfüllt. Die chemische Analogie ist bei $\mathrm{NaCl}_{(35)}$ und $\mathrm{NaCl}_{(37)}$ zur chemischen Identität gesteigert, da bezüglich der negativ elektronischen Ionhüllsphäre, als dem "Sitz" der chemischen Verhältnisse, Gleichbeit be-teht. DaB die zwei Komponenten die Fähigkeit haben, in jedem Verhältnis zusammenzukristallisieren, kann nicht zweifelhaft sein, wenn auch nur ein einziges Mischungsverhältnis bislang gefunden ist ${ }^{2}$ ), ebenso daB Kristalle $\mathrm{NaCl}_{(35)}$ und $\mathrm{NaCl}_{(37)}$ gleiche Kristallform zeigen würden.

2. Die Anordnung der Natrium- und Chlorteilchen im Steinsalz

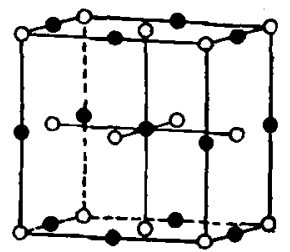

Fig. 1. Stereogramm des Steinsalzes. Nach W. H. und W. L. Bragg.

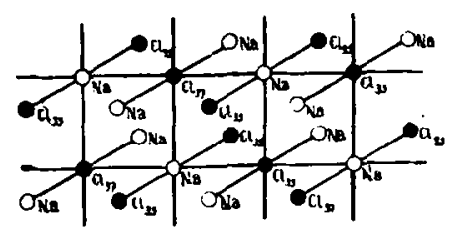

Fig. 8. Isotop-isomorpher Mischkristall $\mathrm{NaCl}_{(35,37)}$ ist als eine Kompromißstellung zufolge des lokalisierenden Einflusses anziehender und abstoßender $\mathrm{Kräfte}$ anzusehen. Demnach ist jeder Atomart ein "Bereich" zuzuschreiben, den sie gewissermaßen als Raumreservat für sich in Anspruch nimmt und nach Möglıchkeit von anderen

1) Auch geringe Beimengungen von $\mathrm{Cl}_{(39)}$ werden angenommen.

2) Es wäre zu untersuchen, ob Steinsalz sehr verschiedenen geologischen Alters, etwa der Lagerstätten des Zechsteins und des Tertiärs, oder Materialien stark abweicbender Entstehungsart, wio solche eruptiv-fumaroler und solche sedimentärer Natur, gleiche oder verschiedene Isotopenmischung aufweisen
Teilchen freibält. Diese „Atombereiche“ fallen also weder mit der "körperlichen" Grenze der Atome zusammen, die durch die Bahnen der außeren Elektronen markiert wird, noch mit seiner "Wirkungssphäre" im eigentJicben Sinne des Wortes; die "Wirkung" anziehender Kräfte geht naturgemäß aber den jeweiligen Kompromißabstand der Teilchen hinaus und ist über mehrere Raumgitterperioden ausklingend zu denken.

Terfahrt man im Sinne von W. L. Bragg ${ }^{3}$ ) durch Ubertragen der Dimensionen von Atombereichen kristallisierter Elemente auf den Feinbau von Verbindungen bei Voraussetzung dichter Packung, unter Vergleich und Ausgleich der so gefundenen Maße, so lassen sich auf

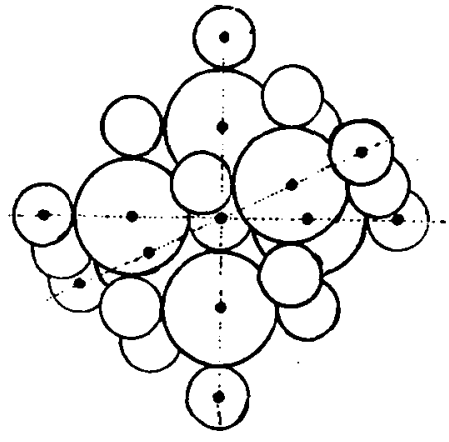
achsialer Zeichnung.
Fig. 3. Atombereiche des Steinsalzes in

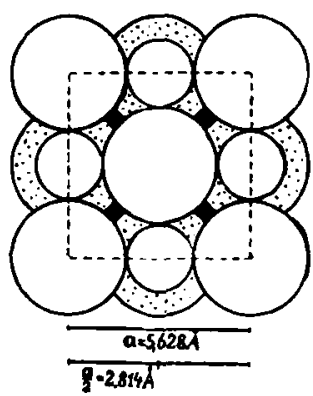

Fig. 4. Atombereiche und Kristallschächte des Steinsalzes im Würfelanblick.
Grund einer Neuberechnung folgende Zablen in Ångstro m einheiten $\left(10^{-8} \mathrm{~cm}\right)$ für den Durchmesser d der Bereiche neutraler und ionistischer Atome der Alkalien, sowie für die Halogene aufstellen ${ }^{4}$ ).

$$
\begin{array}{lll}
\mathrm{Li} 3,02 & \mathrm{Li}^{+} 3,00 & \\
\mathrm{Na} 3,72 & \mathrm{Na}^{+} 3,51 & \mathrm{~F}^{-} \mathbf{1 , 1 7} \\
\mathrm{K} 4,59 & \mathrm{~K}^{+} 4,15 & \mathrm{Cl}^{-} 2,12 \\
\mathrm{Rb} 4,94 & \mathrm{Rb}^{+} 4,47 & \mathrm{Br}^{-} \mathbf{2 , 4 5} \\
\text { Cs } 5,37 & \mathrm{Cs}^{+} 5,04 & \mathrm{~J}^{-} \mathbf{2 , 9 3}
\end{array}
$$

Hinzugefugt seien noch d-Ausmaße für den chemisch zwiespāltigen Wasserstoff, dessen Bereich im neutralen oder positiven (alkaliscben) Falle nach Amin of ${ }^{5}$ ) voraussichtlich den beträchtlichen Durchmesser $\mathrm{d}=$ etwa $1,6-2,2 \AA$ A besitzt; für $\mathrm{H}$ leitet sich unter Benutzung der Ausmaße der $\mathrm{Li}^{+} \mathrm{H}^{-}-$Zelle$^{6}$ ) der sehr kleine $\mathrm{d}$-Wert von $1,10 \cdot 10^{-8} \mathrm{~cm}$ ab.

Dementsprechend ist für das Chlornatrium die Fig. 3 mit den Ausmaßen der hier achsial angeordneten Atombereiche $\mathrm{d}_{\mathrm{Na}}+=3,51$ und $\mathrm{d}_{\mathrm{Cl}}-=2,12$ nach der Formel $\mathrm{Na}\left(\mathrm{Cl}_{6}\right) \mathrm{Cl}(\mathrm{Na})_{6}$ gezeichnet. Den Anblick eines Steinsalz-Elementarwürfels von vorn hat man in Fig. 4. In Ansehung der Kantenlänge $a=5,628 \cdot 10^{-8} \mathrm{~cm}$ einer würfeligen Steinsalzzelle beträgt das Volum dieses Elementarkorpers $178,26 \cdot 10^{-24} \mathrm{ccm}$. Auf $1 \mathrm{~mm}$ Länge sind 1,78 Millionen Würfelchen gereiht, und auf $1 \mathrm{ccm}$ kommen 5600 Trillionen mit 1400 Trillionen Molekülen. Vom Volumen des Elementarkörpers nehmen die Atombereiche 110,53. $10^{-24} \mathrm{ccm}$ in Anspruch (90.57 für $\mathrm{Na}^{+}$und $19,96 \mathrm{für} \mathrm{Cl}^{-}$), so daß ein freier Raum von $57,73 \cdot 10^{-24} \mathrm{ccm}$ verbleibt. Die Prozentzahl für diese Porosität der Steinsalzkristalle ist somit 38.

3. Zur näberen Kennzejchnung verschiedener Ausschnitte des Baues seien hier die Figuren 4-8 verglichen. Sie stellen das Aussehen des Steinsalzdiagramms auf den Flächen des Würfels, Rhombendodekaeders, Oktaeders und des Pyramidenwürfel (210) dar. Es handelt sich ùm Grundrisse entsprechend den jeweiligen Medianschnitten durch dit kugeligen Atombereiche. Es gelten für sie folgende Zahlen:

Fläche in $10^{-16} \mathrm{qcm}$ Anteil in Lük- Ver-

Fläche in $10^{-16} \mathrm{qcm} \quad \begin{aligned} & \text { Anteil in } \\ & 10^{-16} \mathrm{qcm}\end{aligned} \quad$ Lücke $\underset{\text { in } \% \text { kigkit }}{\text { hält- }}$

1. Würfelbezirk $31,67\left\{\begin{array}{lr}2 \mathrm{Na} & 19,35 \\ 2 \mathrm{Cl} & 7,05\end{array}\right\} 26,41 \quad 5,2516,62 \quad 1$

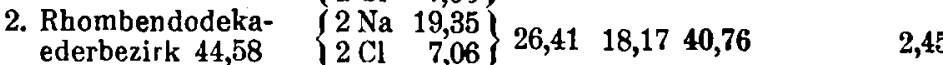

3. Oktaederbezirk $\left\{\begin{array}{ll}2 \mathrm{Na} & 19,35\end{array}\right\} 19,35 \quad 7,8128,76$

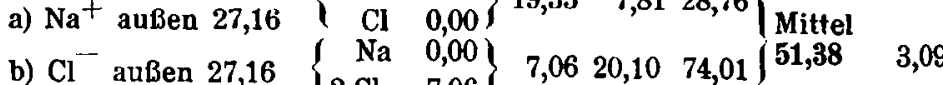

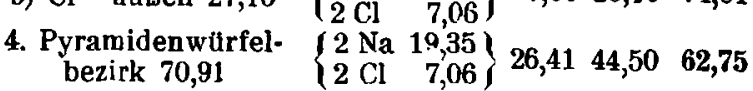

3) W. L. Bragg, Phil. Mag. 40, 169 [1920].

4) F. Rinne, Zeitschr. f. phys. Chemie, 100, 408 [1922].

3) G. A minoff, Meddel. Stockholms Högsk. Min. Inst. Geol. Fören, 43 389 [1921].

6) J. H. Bijvoet a. A. Korssen, Akad. Wetensch. Amsterdam, 31, 49; Ref. Chem. Zentralbl., Bd. 1, 319 [1922]. 
Nach der Tiefe zu folgen die Ionenpacken für die in Rede stehenden Flächen in den $10-8 \mathrm{~cm}$-Abständen 2,$814 ; 1,99 ; 1,624 ; 1,259$.

Ein Abschluß im Grundriß durch die Atombereiche macht sich beim Aufblick auf die Oktaeder- und Pyramidenwïrfelflächen (Fig. 6, 7,8) geltend; mit der ersten bis dritten bzw. ersten bis vierten Lage der Ionenpacken ist der gesamte Grundriß beim Oktaeder bedeckt, beim Pyramiden würfel mit der fünften. Beim Würfel und Rhombendodekaeder hingegen liegen diese Verhältnisse insofern sehr eigenartig, als hier ein voller Abschluß überhaupt nicht zustande kommt; vielmehr bleiben auf; sie zeigt, daß die Berührung von Atombereichen positiv und negativ geladener Ionen bei dieser Bewegung erhalten bleibt. Dem Einwand von G. Tammann ${ }^{10}$ gegenüber J. Stark, daß nicht auch Translation nach den Oktaederflächen statthat, obwohl ihnen jeweils positive und negative Ionenpacken parallel gehen, kann man wohl in Ansebung der allgemeinen Verhältnisse der Verschiebungsmöglichkeit von Bauteilen in Kristallen begegnen; es spielt hier der Ebenenabstand der Bauschichten eine Rolle, dessen Verengung mitbedingend ist für die Erschwerung einer Lagenveränderung. Dieser Abstand beträgt, wie

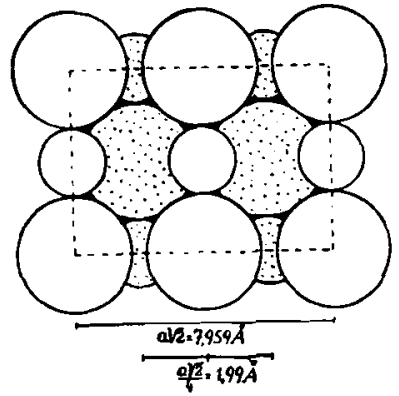

Fig. 5. Atombereiche und Kristallschächte des Steinsalzes in Rhombendodekaederanblick.

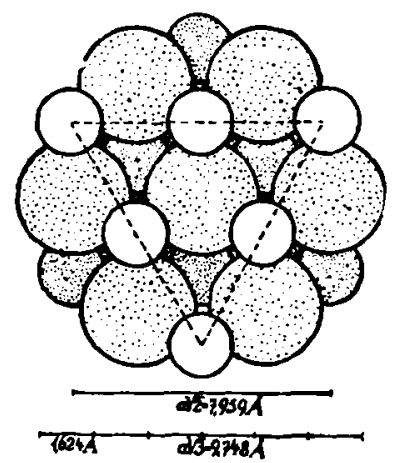

Figg. 6 u. 7. Atombereiche de

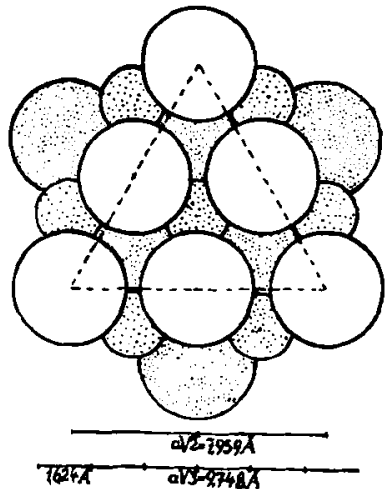

Steinsalzes im Oktaederanblick.

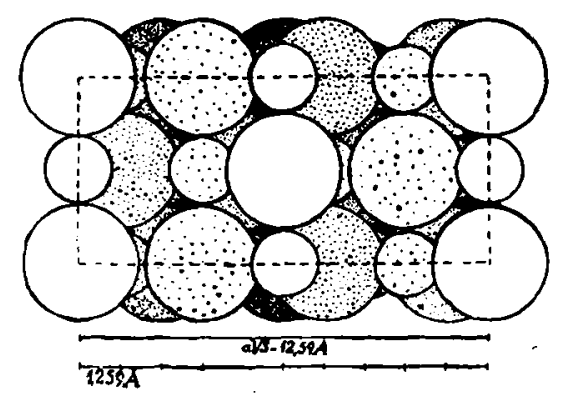

Fig. 8. Atombereiche des Steinsalzes im Pyramidenwürfelanblick.

durch den ganzen Kristall hindurchziehende feinbanliche "Schächte" ubrig. In den Figg. 4 und 5 sind sie schwarz vermerkt.

4. Die physikalischen und chemischen Verhältnisse des Steinsalzes hängen naturgemäß mit seinem Feinbau eng zusammen. In dem Sinne mögen einschlägige Erörterungen als Anregungen wirken.

So lassen sich die röntgenographisch ermittelten Werte für die Zelle z. B. zum Zwecke der Erkundung des exakten spezifischen Gewichtes einer Substanz, im vorliegenden Falle des Steinsalzes, verwenden. Es gilt die Formel

$$
\mathrm{d}=\frac{\mathrm{n} \cdot \mathrm{MG}}{\mathrm{N} \cdot \mathrm{V}_{\mathrm{z}}}
$$

in der d die Dichte, $\mathrm{n}$ die Zahl der Zellenmoleküle, MG das Molekulargewicht, $\mathrm{N}$ die Loschmidtsche Zahl und $\mathrm{V}_{\mathbf{z}}$ das Zellenvolum bedeutet. Beim Steinsalz ist bei Zimmertemperatur danach

$$
\mathrm{d}=\frac{4 \cdot 58,46}{60,6 \cdot 10^{22} \cdot 178,26 \cdot 10^{-24}}=\frac{4 \cdot 58,46 \cdot 100}{60,6 \cdot 178,26}=2,1646
$$

ein Wert, der sich gegenüber anderen Angaben wohl durch Genauigkeit auszeichnen wird, da sonst bei Bestimmungen des spezifischen Gewichtes leicht vorkommende Fehlerquellen wie Poren und Beimengungen hier nicht einwirken.

Von Interesse ist es auch, die Umstände der Festigkeit des Steinsalzes an Hand der hier aufgestellten Atombereichsfiguren feinbaulich zu betrachten. Es wird durch sie wohl ganz besonders anschaulich, daß die den Zusammenhalt kennzeichnenden Zahlen für dies Material mit der Richtung sehr stark wechseln, wie schon L. Sohncke ${ }^{\text {) }}$ erwies. A. Sella sowie W. Voig ${ }^{8}$ ) fanden bei Prismen, die von Würfelflächen begrenzt waren, bei Beanspruchung in Richtung der Würfelnormale die Zugfestigkeit $571 \mathrm{~g} / \mathrm{qcm}$, bei solchen mit Längserstreckung senkrecht zu einer Rhombendodekaederfläche (Seitenflächen Würfel oder Rhombendodekaeder) 1150 und bei in Richtung der Oktaedernormalen gestreckten Probekörpern (Seilenflächen Rhombendodekaeder und Ikositetraeder) 2150. Im übrigen machte sich zudem ein kristallographischer Wechsel der Seitenflächen bei gleichbleibender Längsrichtung der Versuchsprismen im Ergebnis sebr deutlich geltend: Stäbe mit der Längsrichtung in einer Würfelnormalen und mit Würfelsestenflächen lieferten die Zahl 571, hingegen solche mit Rhombendodekaederseitenflächen den Wert 917. Es zeigt sich also eine ${ }_{\text {"Ha u t- }}$ wirkung“ und ein Wechsel mit der Orientierung dieser Oberflächenzone. Der Vergleich der Fig. 4 und 5 macht das qualitativ durch die großen baulichen Unterschiede dieser Hautarten verständlich; einfache zahlenmäßige Zusammenhänge stehen indes noch aus, wohl ein Zeichen für die verwickelte Art der Verhältnisse.

Die Spaltbarkeit des Steinsalzes nach den Würfelflächen läßt sich nunmehr unter Berücksichtigung der Atombereiche durch die Fig. 9 versinnbildlichen, in der die stark ausgezogene geschläingelte Linie Sp-Sp sich auf die obere, die gestrichelte auf die zwette Alomlage bezieht. Wesentlich wird im Sinne von J. Stark ${ }^{9}$ ) der Umstand sein, daß eine Verschiebung der Ionenpacken längs einer feinbaulich buckeligen Würfelfläche die Atombereiche gleichgeladener Teilchen in Berübrung bringt und damit das Gebäude im Spaltungsvorgange elektrisch zersprengt.

Die Translation genannte Parallelverschiebung von Kristallteilen ohne Aufhebung des Zusammenhaltes tritt beim Steinsalz nach den Flächen des Rhombendodekaeders entsprechend der in Fig. 10 an der Oberfläche der Atombereiche sich hinschiängelnden starken Linie T-T

7) L. Sobncke, Poggendorffs Annal. 13i, 177 [1869].

s) W. Voigt, Kristallphysik 946 [1910]

9) J. St ar k, Jahrb. d. Radioaktivität 12, 292 [1915].

schon erwähnt, für die Würfelflächen $2,814 \AA$, für das Rhombendodekaeder 1,990, für das Oktaeder 1,624, womit für den Steinsalzbau eine Verschiebungsmogglichkeit nicht mehr besteht, wie es scheint wegen der geringeren AusmaBe dieser Entfernung und der entsprechend größeren Intensität der zusammenhaltenden Kräfte.

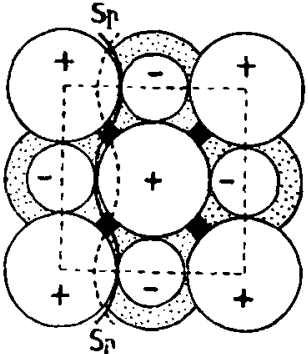

Fig. 9. Atombereiche und Spalt barkeit $\mathrm{Sp}$ des Steinsalzes.

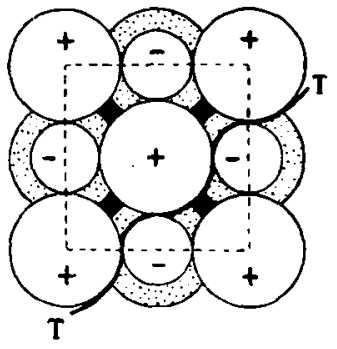

Fig. 10. Atombereiche und Translation T des Steinsalzes.
5. Schließlich ist es jetzt auf Grund der Atombereichsfiguren auch möglich, einige Umstände bezüglich der weiteren Erforschung des Zusammenbanges zwischen feinbaulichen Verhältnissen und den Erscheinungen des Wachstums von Steinsalzkristallen za erörtern.

Die Einsicht in die allgemeinen, ersichtlich komplexen Umstände ist durch die Ergebnisse von Überlegungen seitens $R$. GroB ${ }^{11}$ ), P. Nigg $\left.\right|^{12}$ ) sowie J. J. P. Valeton ${ }^{13}$ ) wesentlich gefördert worden, vom erstgenannten insbesondere durch die geometrische Diskussion der Intensitätskurven des Wachstums, von P. Niggli durch den Hinweis auf die W'ichtigkeit der ungesättigten Oberflächenzone, deren Stärke unter verschiedenen Kristallflächen gesetzmäßig wechselt und damit das $\mathrm{MaB}$ der Ablagerung neuer Teilchen wesentlich mit regelt; J. J. P. Valet on wies auf die sehr verschiedene Anteilnahme positiver und negativer Ionen an der Zusammensetzung von Kristallflächen des Steinsalzes hin, Umstände, die das Festhalten aufprallender Ionen durch den Kristall beeinflussen. Würfel, Oktaeder und Rhombendodekaeder sind in der Hinsicht verschieden gebaut, so daß ihre untereinander abweichenden Wachstumsgeschwindigkeiten feinbaulich verständlich werden, während eine Verschiedenheit der ungesättigten Oberflächenzone unter ihnen hier nicht zur Erklärung dienen kann.

Im Hinblick auf die hier aufgestellten Figg, 4-8 bietet sich in dieser Frage ein neues Moment der Erwägung dar, das zur Erklärung der verschieden geschwinden Stoffablagerung in Richtung der Normalen auf die ungleichen Kristallelächen beitrasen kann. Es ist gewiß eine naturgemäße Vorstellung, daß der Absatz neuer Teilchen auf dem Kristall von dem Grade der Witksamkeit seiner von den Ionen ausgehenden anziehenden Krärte abhängt. In der Hinsicht ist es beachtenswert, daß beim Würfel und Rhombendodekaeder eine Abschirmung schon durch den zweiten Ionenpacken vollzogen ist (Figg. 4 und 5). Die anziehende Wirkung des dritten Packens nach außen auf die Teilchen der Lösung ist durch die äußerste und die zweite Lage gedämpft, während das beim Oktaeder und Pyramiden-

10) G. Tammann, Metallographie, 2. Aufl., 67 [921].

${ }^{11)}$ R. Groß, Abhandl. matb.-phys. Klasse d. Sächs. Ges. d. Wissensch. 35 $137[1918]$

12) P. Niggli, Ztschr. f. anorg. Chem. 110, 55 [1920].

$\left.{ }^{13}\right)$ J. J. P. Valeton, Phys. Zeitschr. 21, 606 [1920]. 
wärfel (Fig. 6, 7 und 8) erst hinsichtlich tieferer Zonen statthat. Entsprechend sind die Wachstumsverhältnisse für die erstgenannten Formen ungünstiger als für die letzteren. In dem Verhältnis von Würfel zu Rhombendodekaeder kommt zu ungunsten des ersteren das größere $\mathrm{MaB}$ des Abstandes seiner Lagen binzu. Unter Mitwirkung solcher Umstände werden die Würfelflächen spärlich versorgt; sie bleiben nahe dem Urpunkt und erscheinen, itn Sinne der Darlegungen von F. Becke ${ }^{14}$ ) und A. Johnsen ${ }^{15}$ ), bei solch kleinbleibenden Zentraldistanzen am Kristall mit großer Flächenentwicklung.

Für den Wechsel der Steinsalz-Kristalltracbt unter dem Einfluß von Lösungsgenossen ${ }^{16}$ ) müssen, wie auch J. J. P. Valeton vermerkte, die R. Marcschen Vorstellungen in Betracht gezogen werden. In reiner Lösung zu virtuellem, starken Normalenwachstum begünstigte, also nicht an Kristall auftretende Flächen (Oktaeder und Pyramidenwürfel) werden voraussichtlich durch Adsorptionen in ihrer Normalengeschwindigkeit des Wachstums gedämpft und damit realisiert.

[A. 175.]

\section{Die Kalisalze im Tier- und Pflanzenkörper, die Grundlage ihrer weltwirtschaftlichen Bedeutung.}

Von P. KRische, Berlin-Lichterfelde.

Es ist mir eine besondere Genugtuung, über die physiologische Bedeulung des Kaliums einen Beitrag für die Ehrengabe zu H e in $r$ i c h Prechts 70. Lebensjahr zu liefern, da unser Altmeister der Kaliindustrie bereits in jungen Jahren dieser Frage seine Aufmerksamkeit zugewandt hat und auf Probleme hinwies, die in der letzten Zeit, wie ich noch ausführen werde, außerordentlich brennend geworden sind.

Die weltwirtschaftliche Bedeutung des Kaliums, die niemals so sichtbar zum Ausdruck kam wie während der vollständigen Umkrempelung der weltwirtschaftlichen Beziehungen während des Weltkrieges, liegt zuletzt in der physiologischen Rolle des Kaliums im Tier- und Pflanzenkörper begründet.

Obwohl wir seit nahezu 100 Jahren bereits durch die Untersuchungen von Pflanzen- und Tieraschen über den Kaligehalt beider unterrichtet sind und soviel wissen, daß die Rolle des Kaliums in beiden nicht unbedeutend ist, so ist es bisher immer noch nicht gelungen, eine deutliche und klare Erkenntnis über die physiologische Rolle des Kaliums im Tier- und Pflanzenkörper zu gewinnen.

Ich kann daher hier nur zusammenfassend berichten über die Arbeiten und Ergebnisse, welche auf diesem Gebiet bisher gezeitigt worden sind, und die Auffassungen verfolgen, die auf Grund dieser Ergebnisse vertreten wurden.

1. Die physiologische Rolle des Kaliums im Tierkörper.

a) Allgeme in es. Über die physiologische Rolle des Kaliums im Tierkörper läßt sich kaum mehr sagen, als daß es durchaus notwendig für jeden normalen Daseinsprozeß organischer Wesen ist, über das einzelne dagegen ist man, abgesehen von wenigen Anläufen zur Erkenntnis, lediglich auf unbestimmte Vermutungen angewiesen. Bekennt doch $0 . \mathrm{Ke} \mathrm{l}\left(\mathrm{ne}^{1}\right.$ ) in seinem grundlegenden Werke: Die Ernährung der landwirtschaftlichen Nutztiere, ganz offen: ,Über die Bedeutung des Kaliuns in de.l Geweben ist nichts sicheres bekannt."

Jedenfalls ist das Kalium ein chemischer Bestandteil des Tierkörpers und findet sich besonders in den Zellgebilden vor, so im blutfreien Muskel, in den Nerven, den Drüsen und Blutkörperchen, auch im Fleischsaft, der Ernährungsflüssigkeit und in der Milch (als phosphorsaures Kalium). In der Muskelsubstanz ist sogar reichlich Kalium enthalten. Nach KeIl ner gibt $1 \mathrm{~kg}$ verbrannter Muskelsubstanz $8 \mathrm{~g}$ Asche, und darin sind 9-10\% Kali. Die Asche von Ochsenblut enthält $60 \%$ Alkalien, von Schafblut $52 \%$, von denen ein Teil als Kali nachgewiesen ist.

Aus diesen Tatsachen geht ohne weiteres hervor, daß dem Kalium bei der Ernährung und dem Stoffwechsel verschiedene wichtige Aufgaben zufallen. Gleich den anderen kohlensauren oder phosphorsauren Alkalien scheint das Kali zufolge seiner chemischen Eigenschaften dahin zu wirken, daß die sich im Körper bildenden Säuren leicht und rasch gebunden werden, so daß sie das Zellenleben nicht besonders beeinträchtigen und ohne störenden Einfluß auf die osmotischen Zellenvorgänge durch die Nieren ausgeschieden werden können. Solche Säuren bilden sich z. B., wenn ein Teil des Nährstoffeiweißes durch Fermente der Bauchspeicheldrüse und durch Bakterien in Stoffe zersetzt wird, die bei der Ernährung nicht weiter verwertet werden. Die bei derartigen Fäulnisvorgängen sich bildenden Stoffe sind bei den Wiederkäuern namentlich Phenol, Kresol,

14) F. Becke, Schriften des Vereins z. Verbreitung naturw. Kenntn. in Wien, 37, H. 15 [1897].

13) A. Johnsen, Vortrag, Vers. d. Naturf. u. Ärzte 1910.

16) Aus reiner Lösung erhält mau Chlornatrium in Würfelform, in einer stark harnstoffhaltigen oder mit Formamid versetzten Lösung Kristalle mit Oktaederflächen, in glykokollführenden Lösungen solche mit Pyramidenwürteln

1) O. Kellner, Die Ernährung der landwirtschaftlichen Nutztiere. 1. Aufl Berlin 1905. Verlag Paul Parey. Jetzt 9. Aufl.
Indol, Skatol usw., die sich gewöhnlich mit Schwefelsäure gepalart als Ätherschwefelsäuren an Kali und Natron gebunden im Harn vorfinden. Daß das Kalium tatsächlich ein unentbehrlicher Nährstoff ist und in beträchtlichen Mengen vom Tierkörper assimiliert wird, beweist auch die Tatsache, daß vom säugenden Jungvieh Kali neben anderen anorganischen Stoffen in den Organismus eingeführt wird. Bei einem Verzehr von 9 l Milch nimmt ein Kalb z. B. $62 \mathrm{~g}$ Gesamtasche auf. Von den $16 \mathrm{~g}$ Kali, welche in dieser Gesamtasche enthalten sind, werden $31 / 4 \mathrm{~g}$, also etwa $21 \%$ des Verzehrs an Kali, angesetzt. Wie unentbehrlich und spezifisch in seinen Funktionen das Kali ist, ergibt sich weiter aus der Tatsache, daß es nicht gelingt, den Kaligehalt der Organe durch das nahverwandte Natron zu ersetzen. Das Mischungsverhältnis der Stoffe, die an dem Aufbau der am Stoffwechselprozeß lebhaft beteiligten Organe mitwirken, ist außerordentlich konstant, besonders z. B. bei der Milchdrüse. Nun enthalten ja alle landwirtschaftlichen pflanzlichen Futterstoffe außerordentlich viel Kali, so daß3 man meinen sollte, es könnte auch bei knapper Fütterung nie an dem nötigen Kali fehlen. Hier ist aber zu beachten, daß der größte Teil des assimilierten Kalis lediglich als Durchgangsstoff dient und schnell wieder ausgeschieden wird, einmal, wie oben erwähnt, an Säuren gebunden, weiterhin als Chlorkalium. G. $B$ ung e ${ }^{2}$ ) hat schon 1873 die Vermutung ausgesprochen, daB der Kalireichtum der Pflanzen die Ursache des hohen Kochsalzbedürfnisses der Pflanzenfresser sei. "Wenn nämlich ein Kalisalz, z. B. kohlensaures Kalium, in wässeriger Lösung mit dem Chlornatrium zusammentrifft, so findet eine teilweise Umsetzung statt; es bildet sich Chlorkalium und kohlensaures Natron. Nun ist bekanntlich Chlornatrium der Hauptbestandteil unter den anorganischen Salzen des Blutplasmas. Wenn also Kalisalze durch Resorption der Nahrung in das Blut gelangen, so eriolgt auch dort jene Umsetzung. Es bildet sich Chlorkalium und das Natronsalz der Säure, die an das Kali gebunden war. Statt des Chlornatriums enthält das Blut jetzt ein anderes, seiner Zusammensetzung nicht angehöriges Natronsalz. Es ist ein fremder Bestandteil oder ein Überschuß eines normalen Bestandteils - z. B. kohlensaures Natron -- im Blute aufgetreten. Die Niere aber hat die Funktion, die Zusammensetzung des Blutes konstant zu erhalten, also jeden abnormen Bestandteil und jeden Ưberschuß eines normalen auszuscheiden. Deshalb wird das gebildete Natronsalz zugleich mit dem Chlorkalium durch die Niere ausgeschieden, und das Blut ist an Chlor und Natron ärmer geworden. Dem Organismus ist also durch Zufuhr von Kalisalzen Kochsalz entzogen worden. Dieser Verlust kann nur durch Wiederersetzung von außen gedeckt werden. Es erklärt sich daraus, daß Tiere, die von kalireicher Nahrung leben, ein Bedürinis nach Kochsalz haben." Anderseits geht aber daraus hervor, da $\beta$ ein reichlicher $Z$ uschuß von Kochsalz einen Kalimangel hervorrufen kann. Das kann sich unter Umständen bei der Ernährung geltend machen, denn die phosphorsäurehaltigen Albuminate, die Bildner der Muskelsubstanz, werden, wie Professor D ü n k e l b e r g in seinem sehr interessanten Buche „Die Grasweide“. angibt (Paul Parey, Berlin 1905) vom Tier nur assimiliert, wenn genügend Kalisalze in aufschließbarer Form in der Nahrung enthalten sind.

Auch beim Stoffwechsel der tätigen Muskeln spielt das Kali eine gewichtige Rolle, ist also nicht unwesentlich an der Produktion von lebendiger Kraft beteiligt. Wenn der Muskel in anhaltende Tötigkeit versetzt wird, so gerät er bald in einen Zustand geringerer Leistungsfähigkeit, er ermüdet. Die Ursache dieser Ermüdung ist in der Ansammlung von verschiedenen Umsatzprodukten zu finden, sogenannten Ermüidungsprodukten, zu denen neben Phosphorsäure, Milchsäure und Kohlensäure auch die Kalisalze gehören.

W. $M$ a y e ${ }^{3}$ ) führt die tierphysiologischen Versuche von $L$ öb an, nach denen die im Pflanzen- und Tierkörper zirkulierenden Lösungen physiologisch im Gleichgewicht stehen. Eine Lösung von Chlornatrium ist $\mathrm{z}$. B. für die Organismen giftig, wird aber durch $\mathrm{Zu}$ führung von Chlorkalium entgiftet.

Jedes Kation oderAnion für sich übt demach eine Giftwirkung aus. Ihre Giftwirkungen heben sich gegenseitig auf undentgiftendie Lösung.

b) Versuche von Urbeanu, Bukarest. Nach der Auffassung von Dr. Urbea n u ${ }^{*}$ ) besteht die ,wunde Seite unserer Ernährungslehre" darin, daß sie sich einseitig auf die Beschaffung von Eiweiß, Fett und Kohlehydraten beschränkt, wobei noch die Eiweißbeschaffung übergebührlich bewertet wird, und daß sie die ebenso wichtige Beschaffung der mineralischen Nährstoffe vernachlässigt. Schon vor 30 Jahren, auf dem ersten rumänischen Kongreß für Medizin, im Jahre 1884 in Bukarest, hat Dr. U r be a $\mathbf{n} u$ in dem Vortrag: „A thiologie der Pellagra, vom chemischen Standpunkt aus betrachtet", die physiologische Notwendigkeit der mineralischen Nährstoffe im allgemeinen, der Kaliumsalze im besonderen betont, den Mangel der letzteren in der üblichen Ernährungsweise des rumänischen Bauers hervorgehoben und ihn in eine enge, kausale Verbin-

अ) G. Bunga, Zeitschr. f. Biol. 1873, 104; 1874, 110; Lehrbuch der pbysiol. u. pathol. Chem. 1898 .

3) W. Mayer. Nenere Ansichten über die Rolle des Kaliums im Pflanzenund Tierbörper. Zeitschr. f. angew. Chem. 1921, Aulsatzteil, S. 589.

4) Prof. Dr. Urbeanu, Die Gefahr einer an Kaliumperbindungen zu armen Ernährungsweise und ibre Beziehung zu Ernährungskrankheiten. Verlag Urban \& Schwarzenberg, Berlin, 1916, broschiert M 5. 